\title{
Isotopic Composition of Presolar Spinel Grain OC2: Constraining Intermediate-Mass Asymptotic Giant Branch Models
}

\author{
Maria Lugaro* \\ Sterrenkundig Instituut Utrecht \\ Postbus 800003508 TA Utrecht, The Netherlands \\ E-mail: M.Lugaro@phys.uu.nl
}

Amanda I. Karakas

Origin's Institute, McMaster University

Hamilton ON, Canada

E-mail: karakas@physics.mcmaster.ca

\section{Larry R. Nittler and Conel M. O'D. Alexander}

Carnegie Institution of Washington, Department of Terrestrial Magnetism

Washington DC 20015, USA

E-mail: Irnedtm.ciw.edu

\section{Christian lliadis}

Department of Physics and Astronomy, University of North Carolina

Chapel Hill, NC 27599-3255, USA

E-mail: iliadis@unc.edu

\section{John C. Lattanzio}

Centre for Stellar and Planetary Astrophysics, School of Mathematical Sciences,

Monash University, Victoria 3800, Australia

E-mail: John.Lattanzio@sci.monash.edu.au

We analyze the $\mathrm{O}, \mathrm{Mg}, \mathrm{Al}, \mathrm{Cr}$ and $\mathrm{Fe}$ compositions predicted by detailed models of AGB stars of different masses and metallicities and discuss them in the light of the precise measurements of the composition of a single extraordinary presolar spinel grain, named OC2. Large excesses of the heavy $\mathrm{Mg}$ isotopes are present in this grain and thus an origin from an intermediate-mass (IM) asymptotic giant branch (AGB) star was previously proposed for it. Our IM-AGB models with temperatures at the base of the convective envelope $\simeq 80-85$ million degrees produce a good match to the composition of $\mathrm{OC} 2$ within the uncertainties related to reaction rates. This solution is possible if, in particular, we take the lower limit and the upper limit for the ${ }^{16} \mathrm{O}(p, \gamma){ }^{17} \mathrm{~F}$ and the ${ }^{17} \mathrm{O}(p, \alpha){ }^{14} \mathrm{~N}$ reaction rates, respectively.

International Symposium on Nuclear Astrophysics - Nuclei in the Cosmos - IX

June 25-30 2006

CERN, Geneva, Switzerland

\footnotetext{
*Speaker.
} 


\section{Introduction}

Presolar grains are extracted from primitive meteorites and analyzed by various microanalytical techniques. Their isotopic compositions are extremely anomalous compared to those found in the bulk of materials formed in the Solar System revealing the stellar origin of these grains [1]. We focus in this paper on the composition of a single extraordinary presolar spinel $\left(\mathrm{MgAl}_{2} \mathrm{O}_{4}\right)$ grain, named OC2 (Fig. 1). Its most remarkable feature is large excesses of the heavy $\mathrm{Mg}$ isotopes; ${ }^{25} \mathrm{Mg} /{ }^{24} \mathrm{Mg}$ and ${ }^{26} \mathrm{Mg} /{ }^{24} \mathrm{Mg}$ are enriched with respect to solar composition by $43 \%$ and $117 \%$, respectively. The ${ }^{17} \mathrm{O} /{ }^{16} \mathrm{O}$ ratio of $\mathrm{OC} 2$ is $(1.25 \pm 0.07) \times 10^{-3}$, i.e. 3.3 higher than solar, and its ${ }^{18} \mathrm{O} /{ }^{16} \mathrm{O}$ ratio is $(6.94 \pm 1.34) \times 10^{-5}$, i.e. 26 times smaller than solar. The $\mathrm{Cr}$ and the ${ }^{57} \mathrm{Fe} /{ }^{56} \mathrm{Fe}$ isotopic ratios are solar within error bars, with hints of possible small excesses of ${ }^{57} \mathrm{Fe}$ and ${ }^{54} \mathrm{Cr}$.

The highly unusual isotopic composition of OC2, mainly its ${ }^{25} \mathrm{Mg}$ and ${ }^{26} \mathrm{Mg}$ excesses, led Zinner et al. [2] to propose a site of origin for this grain different from that assigned to the majority of presolar oxide grains. The $\mathrm{O}, \mathrm{Mg}$ and $\mathrm{Al}$ isotopic compositions of the vast majority of presolar oxide grains indicate that they originated in red giant and low-mass (LM) asymptotic giant branch (AGB) stars $\left(<3 M_{\odot}\right)$. In contrast, the unique composition of grain OC2 has been tentatively attributed to the nucleosynthesis occurring in an intermediate-mass (IM) AGB star, $\simeq 4$ - $7 M_{\odot}$.

We analyze the $\mathrm{O}, \mathrm{Mg}, \mathrm{Al}, \mathrm{Cr}$ and $\mathrm{Fe}$ compositions predicted by detailed models of IM-AGB stars of different masses and metallicities and discuss them in the light of the precise measurements of the composition of grain OC2. We compute the evolution of stars of different masses and metallicities using the Monash version of the Mt. Stromlo Stellar Structure code. All models include mass loss on the AGB according to the prescription of [3], show deep third dredge-up (TDU) and hot bottom burning (HBB). We performed detailed nucleosynthesis calculations using a postprocessing code which includes 74 species, 506 reactions and time-dependent diffusive mixing in all convective zones. References and details on the evolutionary code, post-processing code, network and reaction rates are outlined in [4]. A more extended version of this paper can be found in [5].

\section{Results}

In Fig. 2 the $\mathrm{Mg}$ composition of grain OC2 is compared to that predicted by our IM-AGB models. Note that the predicted ${ }^{26} \mathrm{Mg} /{ }^{24} \mathrm{Mg}$ ratio includes both the abundance of ${ }^{26} \mathrm{Mg}$ itself and the contribution from ${ }^{26} \mathrm{Al}$ decay. The main process producing ${ }^{26} \mathrm{Al}$ in IM-AGB stars is HBB. In fact, this nucleus is also produced in the He inteshell by H-burning, but is completely destroyed by neutron captures during the recurrent episodes of convective He burning (thermal pulses). Only a very thin region, of the order of $10^{-4} M_{\odot}$, at the top of the intershell is not involved in the convection, and hence ${ }^{26} \mathrm{Al}$ in that region is saved and carried to the surface by TDU. However, the importance of this region is very minor compared to the effect of HBB. It appears that a narrow range of $\mathrm{HBB}$ temperatures is required in order to produce enough ${ }^{26} \mathrm{Al}$ to match the excess at mass 26 shown by OC2 at the given $\delta\left({ }^{25} \mathrm{Mg} /{ }^{24} \mathrm{Mg}\right)$ value, avoiding an increase of the $\delta\left({ }^{25} \mathrm{Mg} /{ }^{24} \mathrm{Mg}\right)$ value itself. This occurs in the models of $5 M_{\odot}$ and $Z=0.008$ metallicity and $6.5 M_{\odot}$ and $Z=0.02$ metallicity (in this latter case within the uncertainties associated to the ${ }^{22} \mathrm{Ne}+\alpha$ and ${ }^{25} \mathrm{Mg}(p, \gamma){ }^{26} \mathrm{Al}$ reaction rates). For the $5 M_{\odot} Z=0.02$ case the temperature at the base of the convective envelope 


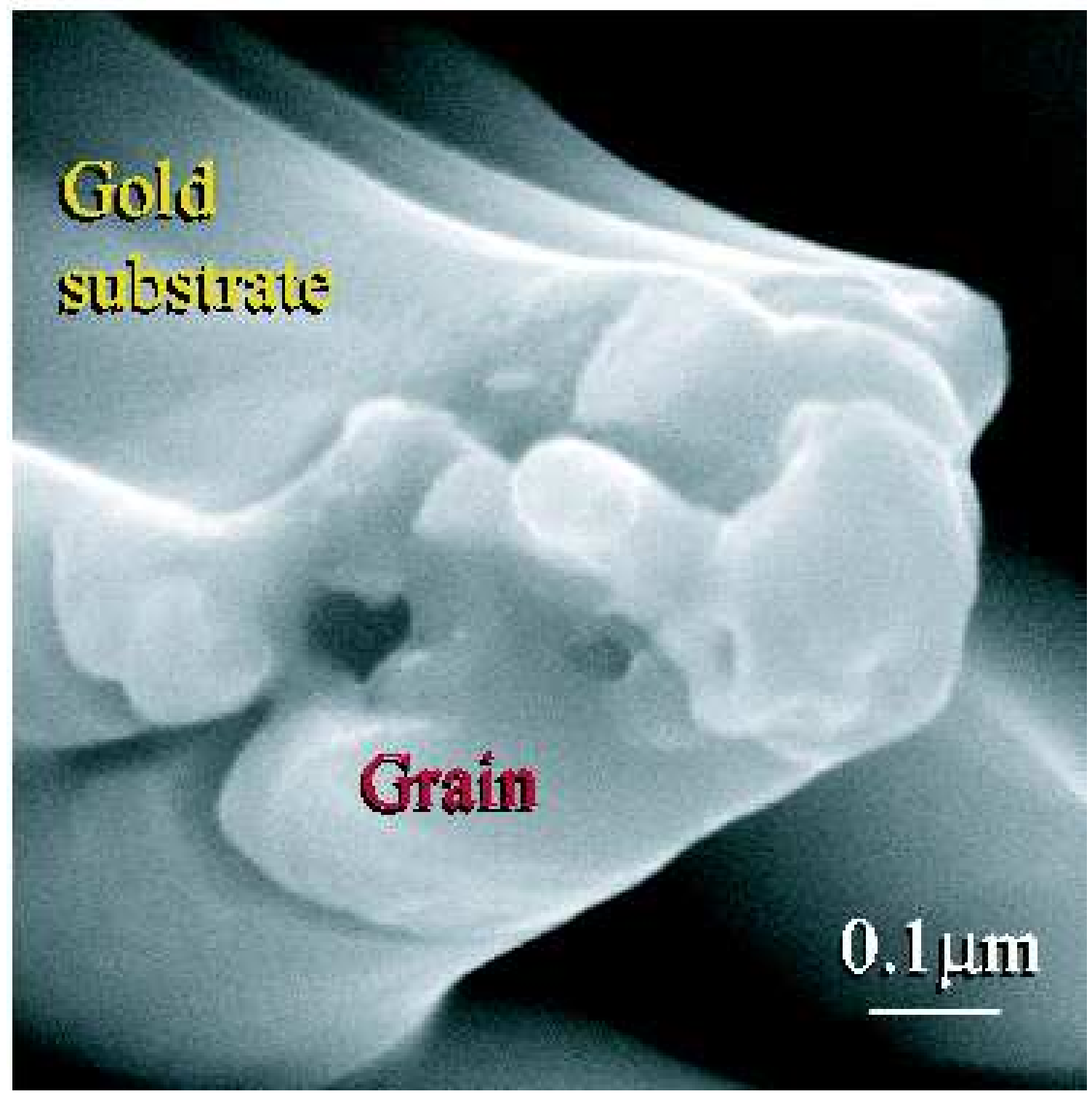

Figure 1: Scanning electron microscope image of presolar spinel grain OC2. This $800 \mathrm{~nm}$ ruby-like gem is sitting on a gold pedestal, following the ion probe isotopic analysis, because the gold substrate sputters faster than the grain does.

is too low to produce ${ }^{26} \mathrm{Al}$ via the reaction ${ }^{25} \mathrm{Mg}(p, \gamma){ }^{26} \mathrm{Al}^{g}$, while in the $6.5 M_{\odot} Z=0.012$ case the temperature at the base of the convective envelope is too high so that also ${ }^{25} \mathrm{Mg}$ is produced by HBB.

In Fig. 3 the ${ }^{17} \mathrm{O} /{ }^{16} \mathrm{O}$ ratio is plotted as function of the $\delta\left({ }^{25} \mathrm{Mg} /{ }^{24} \mathrm{Mg}\right)$ value. The ${ }^{17} \mathrm{O} /{ }^{16} \mathrm{O}$ ratio of grain OC2 is not matched by any of the models, as they always produce too high a ratio. The ${ }^{17} \mathrm{O} /{ }^{16} \mathrm{O}$ ratio during proton captures is determined by the ratio of the ${ }^{16} \mathrm{O}(p, \gamma){ }^{17} \mathrm{~F}$ and the ${ }^{17} \mathrm{O}(p, \alpha){ }^{14} \mathrm{~N}$ reaction rates. This ratio reaches a minimum of $\simeq 0.0011$ around 50 million degrees and then increases again for higher temperatures reaching 0.008 at 100 million degrees (see Fig. 8 of [6]). On the basis of the latest available information [7], we have re-evaluated the ${ }^{17} \mathrm{O}(p, \alpha){ }^{14} \mathrm{~N}$ 


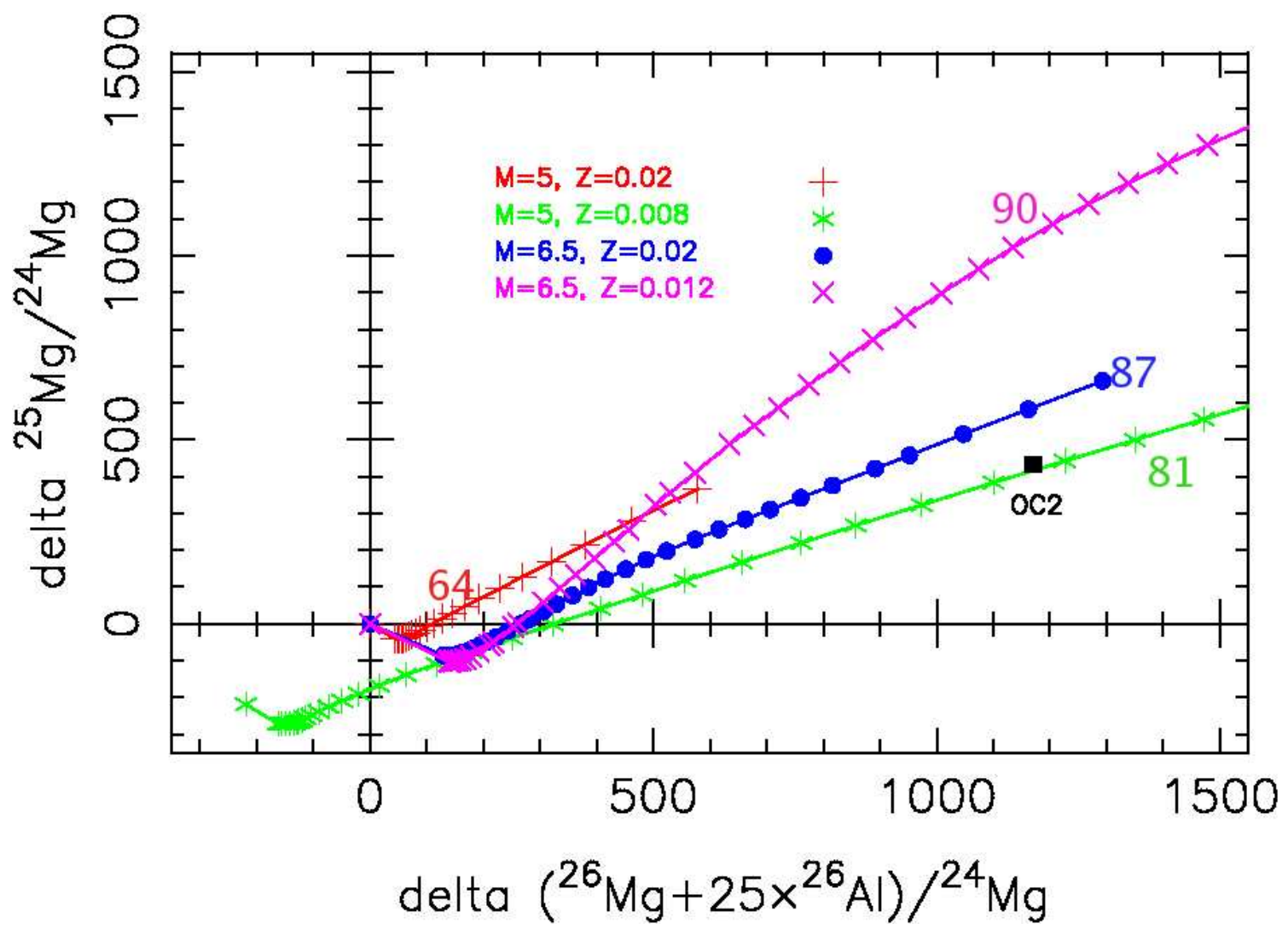

Figure 2: The Mg compositions of grain OC2 is compared to our models of IM-AGB stars. $\delta$ values represent permil deviations from the Solar System ratios. The $2 \sigma$ uncertainties for OC2 are roughly within the symbol. Each symbol for model predictions represents the envelope composition after a TDU episode. Number labels associated to each line of model predictions indicate the maximum temperature (in million $\mathrm{K}$ ) achieved at the base of the convective envelope for each model. As indicated in the x-label the $\delta\left({ }^{26} \mathrm{Mg} /{ }^{24} \mathrm{Mg}\right)$ measured in $\mathrm{OC} 2$ is compared to predictions calculated by including the abundance of ${ }^{26} \mathrm{Al}$ multiplied by a factor of 25 to take into account the fact that $\mathrm{Al}$ is preferentially included in spinel by such factor, given that stoichiometric spinel by definition is $\mathrm{MgAl}_{2} \mathrm{O}_{4}$, i.e. it has $\mathrm{Al} / \mathrm{Mg}=2$, while this ratio is 0.079 in the Solar System. Solar ratios are indicated by ticked axis at $\delta=0$.

rate for this reaction and found a recommended rate close to NACRE with an uncertainty range of $\simeq+25 \%$ and $-30 \%$, for HBB temperatures. For the ${ }^{16} \mathrm{O}(p, \gamma){ }^{17} \mathrm{~F}$ rate NACRE gives uncertainties $\simeq+30 \%$ and $-43 \%$. By combining the upper limit of the ${ }^{16} \mathrm{O}(p, \gamma){ }^{17} \mathrm{~F}$ rate together with the lower limit of the ${ }^{17} \mathrm{O}(p, \alpha){ }^{14} \mathrm{O}$ rate we can find a solution for the ${ }^{17} \mathrm{O} /{ }^{16} \mathrm{O}$ ratio of $\mathrm{OC} 2$, using the same models $\left(5 M_{\odot}, Z=0.008\right.$ and $\left.6.5 M_{\odot}, Z=0.02\right)$ that match its $\mathrm{Mg}$ composition.

As for the ${ }^{18} \mathrm{O} /{ }^{16} \mathrm{O}$ ratio, because of $\mathrm{HBB}$, all the models predict ratios of the order of $10^{-6}$ $-10^{-7}$, thus it is not possible to match the observed value by any model. However, there is always surface contamination on sample mounts and residual oxygen in the ion microprobe vacuum system. The very low measured ${ }^{18} \mathrm{O} /{ }^{16} \mathrm{O}$ ratio for grain $\mathrm{OC} 2$ was based on 35 actual counted ${ }^{18} \mathrm{O}$ atoms. If the grain actually had an ${ }^{18} \mathrm{O} /{ }^{16} \mathrm{O}$ ratio of zero, this low measured ${ }^{18} \mathrm{O}$ signal would correspond to a $2 \%$ level of terrestrial contamination, which is perfectly reasonable. Thus, we consider it likely that the true ${ }^{18} \mathrm{O} /{ }^{16} \mathrm{O}$ ratio of OC2 was indeed lower, and do not consider the mis-match 


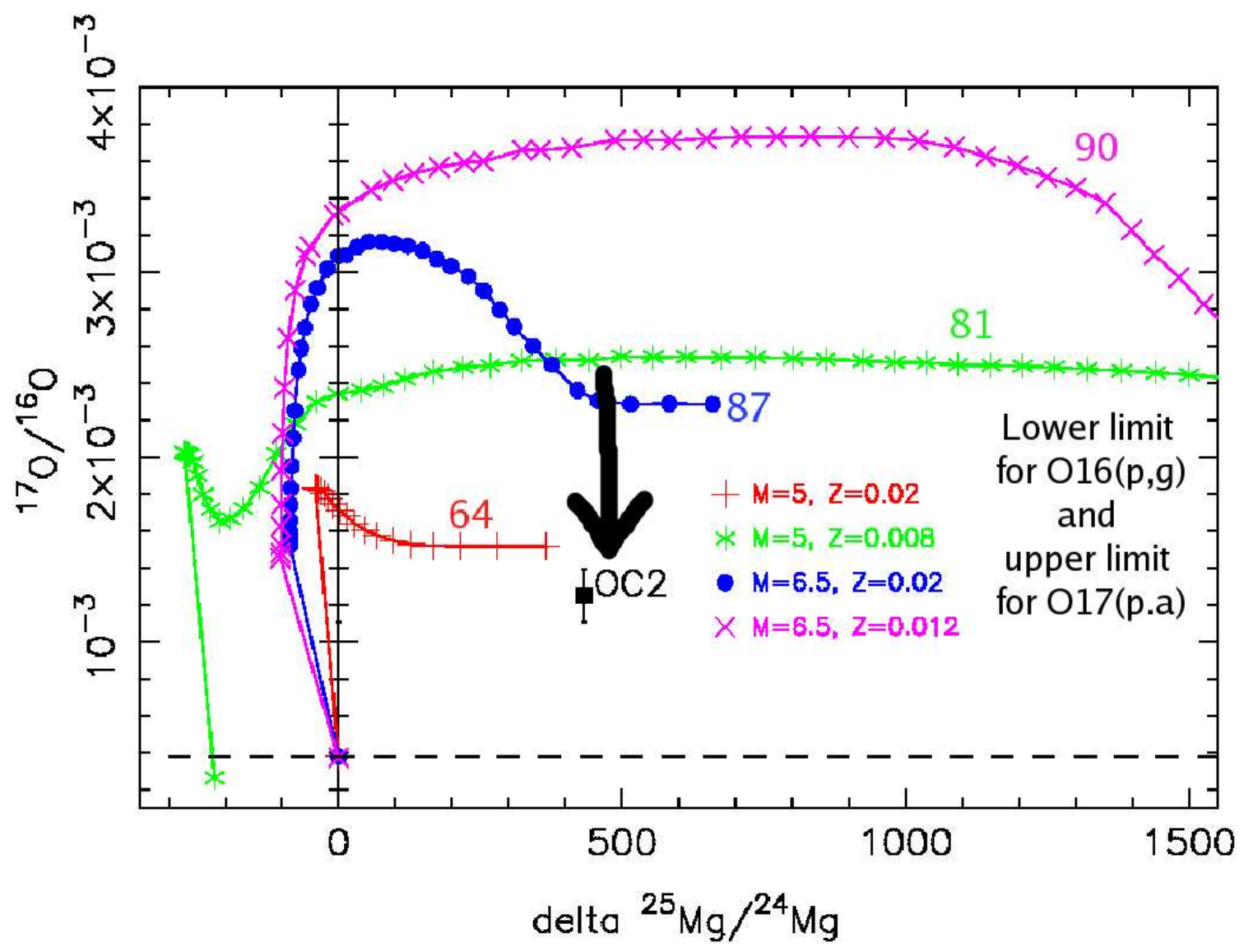

Figure 3: The ${ }^{17} \mathrm{O} /{ }^{16} \mathrm{O}$ ratio is plotted as function of the $\delta\left({ }^{25} \mathrm{Mg} /{ }^{24} \mathrm{Mg}\right)$ for our IM-AGB models. The thick black arrow indicates that the ${ }^{17} \mathrm{O} /{ }^{16} \mathrm{O}$ ratio becomes $\simeq 2$ times smaller, thus matching the composition of $\mathrm{OC} 2$, when using together the lower and the upper limits for the ${ }^{16} \mathrm{O}(p, \gamma){ }^{17} \mathrm{~F}$ and ${ }^{17} \mathrm{O}(p, \alpha){ }^{14} \mathrm{~N}$ reactions, respectively.

with HBB models to be a major problem.

We note that a LM-AGB star of mass $\simeq 2.5$ to $3.5 M_{\odot}$ and low metallicity, $Z \simeq 0.004$ to $\simeq 0.008$ could also produce the ${ }^{25} \mathrm{Mg}$ excess shown by OC2 because the the maximum temperature at the bottom of a thermal pulse and the TDU efficiency are high enough. In this case we would have to assume that some extra-mixing process at the base of the convective envelope [6] is at work during the $\mathrm{AGB}$ phase, in order to produce ${ }^{26} \mathrm{Al}$ and also match the $\mathrm{O}$ ratios. When considering the Fe composition, LM-AGB stars of low metallicity produce much larger excesses in ${ }^{57} \mathrm{Fe}, \delta\left({ }^{57} \mathrm{Fe} /{ }^{56} \mathrm{Fe}\right) \simeq 400$, than do the IM-AGB models, $\delta\left({ }^{57} \mathrm{Fe} /{ }^{56} \mathrm{Fe}\right) \simeq 80$. Unfortunately, the large uncertainty of the measured $\delta\left({ }^{57} \mathrm{Fe} /{ }^{56} \mathrm{Fe}\right)=170 \pm 190$ prevents us from determining which models represent the best match. On the other hand, the IM-AGB models match the $\delta\left({ }^{54} \mathrm{Cr} /{ }^{52} \mathrm{Cr}\right)$ value measured in OC2 (102 \pm 117$)$, while the LM-AGB of low metallicity model would produce $\delta\left({ }^{54} \mathrm{Cr} /{ }^{52} \mathrm{Cr}\right)$ values close to the $2 \sigma$ upper limit of OC2. Moreover, we note that in low metallicity stars the $\delta\left({ }^{50,53} \mathrm{Cr} /{ }^{52} \mathrm{Cr}\right)$ should be largely negative, instead than around solar as observed in $\mathrm{OC} 2$, because of the effect of the Galactic Chemical Evolution of the Cr isotopes. This represents a hint against a LM-AGB star of low metallicity as the parent star for OC2, even though detailed models 
for the evolution of the Cr isotopes in the Galaxy should be performed to confirm this prediction.

\section{Acknowledgments}

ML gratefully acknowledges the support of NWO through the VENI grant. All computations were performed on Canadian Institute for Theoretical Astrophysics's Mckenzie cluster which was funded by the Canada Foundation for Innovation and the Ontario Innovation Trust. The work of LRN and CA was supported by NASA. This work was partially supported by the Australian Research Council.

\section{References}

[1] M. Lugaro 2005, Stardust from Meteorites: An Introduction to Presolar Grains, World Scientific, Singapore

[2] E. Zinner, et al. 2005 Oxygen, magnesium and chromium isotopic ratios of presolar spinel grains, Geochim. et Cosmochim. Acta, 69, 4149

[3] C. Vassiliadis, \& P. R. Wood 1993, Evolution of low-and intermediate-mass stars to the end of the asymptotic giant branch with mass loss, ApJ, 413, 641

[4] Karakas, A. I., et al. 2006, The Uncertainties in the ${ }^{22} \mathrm{Ne}+\alpha$-Capture Reaction Rates and the Production of the Heavy Magnesium Isotopes in Asymptotic Giant Branch Stars of Intermediate Mass, ApJ, 643, 471 [astro-ph/0601645]

[5] M. Lugaro, et al. 2006, The composition of presolar spinel grain OC2: constraining asymptotic giant branch models, A\&A, accepted

[6] K. M. Nollett, M. Busso, \& G. J. Wasserburg 2003, Cool Bottom Processes on the Thermally Pulsing Asymptotic Giant Branch and the Isotopic Composition of Circumstellar Dust Grains, ApJ, 582, 1036 [astro-ph/0211271]

[7] A. Chafa, et al. 2005, Hydrogen Burning of ${ }^{17} O$ in Classical Novae Phys. Rev. Lett., 95, 1101 [astro-ph/0506500] 